\title{
Algunas observaciones sobre la capacidad de revisión de los adolescentes
}

\author{
AnNa Camps \\ Universidad Autónoma de Barcelona
}

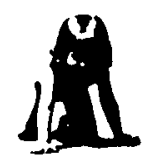

Resumen

Este artículo, a partir de los resultados de las investigaciones sobre el subproceso de revisión y de los modelos más recientes que explican las operaciones implicadas en él, analiza los protocolos de las interacciones orales de un grupo de tres alumnos adolescentes (13.14 años) trabajando en grupo y formula algunas observaciones referidas al tipo de revisiones que efectúan. En esta situación de producción en grupo se pone de manifiesto: a) que los alumnos realizan numerosas operaciones de revisión que en su mayoria no se reflejan en cambios en el texto; b) que dichas revisiones afectan a todos los niveles de producción desde los superficiales a los globales referidos al contenido y a la función del texto y a la situación discuriva; c) que las revisiones pretextuales son fundamentalmente de dos tipos: las que afectan a los planes elaborados oralmente y la que afecta a los que llamaremos texto intentado, o producciones orales formuladas para ser escritas.

Palabras clave: Revisión, Adolescentes, Texto intentado y Texto escrito.

\section{Some observations about adolescents capacity to revise written texts}

\author{
Abstract
}

Based on recent research results on the subprocess of revision and on the latest explaining the operations involved in it, this paper analyses the oral interactions between three adolescent students (13-14 years old) working in group. Furthermore it makes some observations on the type of revisions made by the subjects. In this situation of working in a group, it was found that: a) students carry out many revision operations which on the whole do not effect any changes in the text: b) revisions take place at all levels of production - from superficial to global- relating to the content and function of the text, and to the discurive situation; c) pre-textual revisions fall into two main groups: those affecting orally elaborated plans, and those affecting what be called intended text, i.e., oral productions designed to be written.

Key Words: Revision, Adolescents, Intended text, Written text.

Dirección de la autora: Universidad Autónoma de Barcelona. Departamento de Didáctica de la lengua y la literatura. 
El subproceso de revisión ha sido considerado por muchos autores el más importante dentro del proceso global de la redacción. Murray (1978) aun reconociendo la insuficiencia de las investigaciones efectuadas hasta aquel momento, afirmaba que «escribir es reescribir» (85). Puede considerarse que este subproceso es peculiar del lenguaje escrito y lo diferencia del lenguaje oral. La primera dificultad se halla, sin embargo, en el mismo concepto de revisión y en su definición.

Las investigaciones centradas en el estudio de los cambios que quienes escriben efectúan en sus borradores parecen presuponer que la operación de revisión consiste en modificar aquellos aspectos del texto en los que percibe algún desajuste. Esta es la orientación de muchas de las investigaciones sobre el proceso de revisión que se efectuaron durante los años $70 \mathrm{y}$ principios de los 80 , muchas de las cuales se orientaron a conocer el número de cambios que los autores hacían en sus textos, los niveles a los que revisan y el tipo de cambios que introducían. Las investigaciones de este tipo pretenden en general descubrir diferencias según la edad y el nivel de experiencia de los que escriben. La conclusión más frecuente es que los aprendices efectúan pocos cambios en el texto y cuando lo hacen son de nivel superficial (Emig, 1971; Stallard, 1974; Perl, 1979; Piano, 1979; Sommers, 1979; National Assessment of Educational progress, 1977; Bridwell, 1980). Como señala Hillocks (1986), estos resultados pueden ser el fruto de las investigaciones (límite de tiempo, situación especial de producción, etc.) y que serían, seguramente, diferentes en circunstancias diferentes. Así parecen sugerirlo algunas experiencias como las de Graves (1979) y de Calkins (1985), o investigaciones como la de Gere y Stevens (1985), la cual permite concluir que los aprendices pueden tomar en consideración aspectos de nivel alto, especialmente los referidos a los contenidos temáticos del texto.

Las investigaciones de Nold (1981) y Bartlett (1982) permiten ahondar en la complejidad de los procesos cognitivos que comporta la revisión. Barlett distingue en este subproceso operaciones de detención, de identifica- ción y de corrección, las cuales requieren, según esta autora, capacidades de distinto tipo.

El cambio conceptual más importante lo aporta el modelo de revisión elaborado por Bereiter y Scardamalia $(1983,1987)$, que reconocen también en el proceso de revisión distintas operaciones (comparar, diagnosticar y operar). Estos autores no relacionan la revisión solo con los cambios efectuados realmente en el texto (o borrador) escrito, sino que reconocen la existencia de operaciones de revisión que no desembocan en alteraciones en el texto. Una de las hipótesis que formulan es que el proceso puede aplicarse a lo que quiere escribirse antes de escribirlo; es decir, puede ocurrir que quien escribe intente resolver un desajuste detectado no sobre el texto ya escrito sino en el que tiene intención de escribir a continuación. Witte (1985) destaca que el modelo de Hayes y Flower, al considerar la escritura como un proceso récursivo y jerárquico, contiene en sí mismo implícitos estos conceptos. Según este autor, si se acepta esta caracterización del proceso de composición, tiene que haber revisión ya en el momento de la planificación, por ejemplo. Así pues se puede revisar una frase tanto antes de escribirla (pre-texto) como después. Witte insiste en tres puntos: (1) Los planes del autor pueden concretarse mentalmente como pre-texto, que finalmente puede ponerse por escrito. (2) Tanto la revisión del texto como la del pre-texto pueden estar motivadas de la misma manera, por la percepción de un desajuste, de una disonancia entre las intenciones y lo producido. (3) Ambas revisiones (la del texto y la del pre-texto pueden tener los mismos efectos en el texto escrito. La hipótesis de Witte es que entre la revisión del pre-texto y la del texto ya producido hay una relación inversa. Quienes escriben pueden percibir una disonancia entre sus intenciones y el pre-texto $\left({ }^{\prime} i\right.$ Es realmente esto lo que quiero comunicar?», «Es esta la manera en que quiero comunicar mi mensaje a la audiencia?»). Si la resuelven, no será necesaria la revisión del texto escrito. En cambio, si fracasan, es probable que la disonancia persista en el texto escrito y que haga falta la revisión posterior del mismo. 
Las investigaciones de Matsuhashi (1982, 1987; Matsuhashi y Gordon, 1985) sobre el proceso de composición en «tiempo real» permiten constatar la diversidad de las conductas de revisión según el tipo de texto que se produce. La definición de revisión hasta hacerlo coincidir con el de evaluación o control no sólo del texto escrito, sino también de la representacion mental que de él se hace el escritor:

La revisión es un fenómeno complejo, que se inicia con una serie de impulsos creativos y que refleja no sólo correcciones, sino una constante reevaluación del texto desarrollado y de la representación mental que su autor se hace de él. (Matsuhashi, 1987).

De las conclusiones que se desprenden de las investigaciones de los procesos implicados en la revisión destacaremos tres aspectos:

(1) La jerarquización y recursividad del proceso de escritura implica que la revisión puede darse en momentos y niveles diferentes de la producción escrita.

(2) La revisión no se entiende sólo como cambio en el texto, sino que se considera que el proceso puede llevarse a término sin que desemboque necesariamente en correcciones en el texto que se ha escrito.

(3) La revisión puede afectar a elementos pre-textuales.

En el presente artículo, el análisis de los protocolos orales de un grupo de estudiantes de 13-14 años, que recogen las interacciones producidas en el curso de un trabajo de redacción en grupo, permite algunas observaciones: a) sobre la capacidad de revisión de los adolescentes a partir de algunas preguntas que nos formulamos: ¿son los adolescentes capaces de efectuar operaciones de revisión? ¿a qué niveles revisan? ¿en qué momento? ¿qué desencadena estas operaciones? ¿sobre qué elementos recae la revisión?; b) sobre algunas características del proceso de revisión en grupo, que permite actualizar operaciones que pueden no manifestarse en las situaciones de producción individual o que la propia situación permite que se desencadenen, según la hipótesis de Forman y Garden (1984).

\section{ANALISIS DE LAS OPERACIONES DE REVISION EFECTUADAS POR TRES ALUMNOS EN SITUACION DE PRODUCCION EN GRUPO}

Descripción del trabajo planteado y de las condiciones en que se llevó a término.

Los protocolos que se comentarán son el resultado de los intercambios orales entre tres alumnos de $70^{\circ}$ de EGB durante el proceso de redacción de un texto argumentativo sobre el tema del racismo. El trabajo constituyó la última parte de una secuencia didáctica que tenía por objetivo que los alumnos conocieran las características y la estructura de interpretarlos y producirlos. La secuencia se componía de los pasos siguientes:

1. Propuesta de elaboración de un texto de opinión sobre el tema del racismo.

2. Lectura y comentario de varios textos argumentativos para descubrir su estructura.

3. Análisis detenido de dos de los textos leídos.

4. Información teórica sobre la función y la estructura de un texto argumentativo.

5. Ejercicios orales y escritos sobre algunas estructuras lingüísticas típicas de este tipo de textos: enlaces lógicos, relación entre argumentos y contraargumentos, estructuras concesivas, etc.

6. Visión de un debate televisivo sobre el racismo.

7. Encuesta para recoger distintas opiniones sobre la convivencia entre personas de razas diferentes.

8. Redacción del texto en el que los alumnos debían expresar su opinión sobre el tema, dirigiéndose a una audiencia con el propósito de convencerla.

El trabajo planteado pretendía analizar el proceso seguido por los alumnos cuando escriben para descubrir la posible incidencia que pueden tener en la elaboración de un escrito los conocimientos explícitos sobre las características de un determinado tipo de texto.

El trabajo de redacción se organizó por grupos de dos o tres alumnos con el fin de que tuviesen que verbalizar las operaciones que efectuaban. Aunque la situación de redacción colectiva difiere seguramente en mu- 
chos aspectos de la de redacción individual, se puede considerar que las verbalizaciones obligadas de los alumnos cuando trabajan en grupo reflejan algunas de las operaciones propias del proceso de composición, sin que se infiera de ello que estos mismos alumnos las lleven a término del mismo modo en la escritura individual. En cualquier caso, el hecho de trabajar en grupo hace explicitar las propuestas, las soluciones y los problemas que se plantean y quizás suscita el planteamiento de algunos que no aparecerían en caso de trabajo individual.

La redacción representaba un problema complejo principalmente en cuatro aspectos: (1) los alumnos debían tratar de ajustar su texto a una estructura determinada: la argumentativa; (2) establecer su punto de vista sobre el tema; (3) seleccionar los contenidos entre un número importante de opiniones distintas que habían recogido; (4) tener en cuenta una posible audiencia que no compartiera su opinión. Los protocolos obtenidos ponen de manifiesto que los alumnos de este grupo se plantean el problema de la redacción teniendo en cuenta estos elementos y que el proceso que siguen no es el mismo que el que suelen seguir cuando escriben lo que una alumna de este grupo, Xell ${ }^{1}$, califica de texto «normal», es decir, un texto como los que suele escribir, en que el maestro da unas indicaciones y un posible tema o enunciado y los alumnos escriben, en general de un tirón. Xell lo expresa así:

(1) 134. Xell-—Un text normal! Jo voldría fer un text.

Este comentario puede ser también indicio de que aceptan la tarea escolar que se les impone sin eludirla ni buscar un camino fácil para resolverla. Parece claro que los alumnos de este grupo no siguen un modelo de «decir los conocimientos» (Bereiter y Scardamalia, 1987), sino que se plantean su tarea de manera global, teniendo en cuenta todos los elementos que inciden en ella.

La redacción se planteó como una tarea compleja, justamente para analizar, por una parte, cuáles eran los problemas a que los es- tudiantes se enfrentaban en relación con los contenidos sobre el tema y sobre el tipo de texto, comentados y explicados en clase en sesiones anteriores $y$, por otra parte, para que se explicitaran los problemas y las soluciones planteados. El fundamento de esta decisión era la tesis de Flower y Hayes (1981) según la cual si para resolver la tarea propuesta los alumnos pueden utilizar esquemas que tienen muy interiorizados y en cierto modo automatizados, éstos no aparecerán como reflexión consciente y por lo tanto no serán observables.

El tiempo dedicado a la redacción del texto osciló entre dos horas, que fue el que inicialmente se había destinado a este trabajo, y tres horas, que fue el que necesitaron algunos grupos, entre los cuales el que se presenta en este artículo. Los grupos de trabajo en 7. nivel de EGB fueron 15, cada uno de ellos compuesto de tres y en algunos casos de dos alumnos.

\section{Análisis de los protocolos}

Para poder analizar el proceso se hizo la grabación de los intercambios orales de cuatro de estos grupos. Las observaciones que se presentan en este trabajo toman como base los protocolos orales de uno de los grupos que por sus características y por el tipo de trabajo que llevaron a término es especialmente rico y sugerente. Dichos protocolos están constituidos por 1.034 intervenciones. Para este primer análisis se agruparon las intervenciones en grupos de intervenciones que denominaremos episodios constituidos por secuencias de intervenciones y actuaciones que tienen unidad temática y/o de procedimiento. El cambio de episodio se produce bien por un cambio de tema o bien por un cambio de subproceso. Por ejemplo: los alumnos estan escribiendo y dejan de hacerlo para generar nuevos contenidos, o para discutir la inclusión de algún nuevo elemento temático, o para releer y revisar algún fragmento ya escrito. Por lo tanto cada episodio está caracterizado por la operación dominante que a su vez incluye de manera subordinada diversas operaciones de manera recursiva. 


\section{Algunas observaciones y constataciones}

Transcribimos en primer lugar el texto que escribieron para que sirva de punto de referencia de las discusiones y comentarios de los alumnos en el proceso de producción. Como se podrá ver, en algunas ocasiones los borradores parciales producidos no se recogerán en el texto final que es el siguiente:

(2) El món està ple d'animals que es maten un a l'altre per sobreviure un mateix i mai per diversió ni pel gust de matar, només un animal dels milers que hi ha ho fa. L'home mata per sentir-se superior, per ser el millor i tenir poders.

Quan es van assabentar que hi havien altres cultures va ser quan va començar a aparèixer el racisme, però llavors la nostra ment no estava tan desenvolupada com ara, ja és hora que canviem.

Per què la gent de cultures más baixes estan marginats a la nostra societat? Es veritat que hi ha algunes cultures menys desenvolupades que altres, però per aixó no els hem de rebutjar, sinó ajudar-los; fer escoles, camps de treball, etc...

Us molesta ajudar a les altres races? Si a nosaltres no ens haguessin ajudat encara estaríem al «Neolitic».

Tenen dret a treure'ns el lloc de treball? Aquesta gent que emigren a altres països tenen la mateixa necessitat que nosaltres vam tenir fa uns quants anys, a l'època del franquisme. Per què no els podem ajudar ara?

«El món és de tots $\mathrm{i}$ l'hem de compartir.»

El contraste entre el tiempo dedicado a la redacción y la cantidad de intercambios orales sobre la tarea, por un lado y la brevedad del texto producido, por otro, parece ya un primer indicio de que los intercambios de los alumnos durante el proceso nos pueden decir mucho más sobre la capacidad de redacción de estos alumnos que el texto por sí solo.

A continuación se exponen algunas de las constataciones referidas al proceso de revisión que se desprenden del análisis de estos protocolos.

1 Una primera constatación es que la revisión que hacen los alumnos, cuando consi- deran terminado el primer borrador completo, es muy reducida. Podemos considerar dos momentos:

a) El de "pasar en limpio» el borrador (caótico y desordenado) que han producido. Esta tarea la lleva a cabo una de las alumnas del grupo, Núria, mientras los otros dos miembros del grupo, principalmente Xell, observan y van controlando el trabajo. En este proceso hay operaciones de revisión de algunos aspectos ortográficos desencadenadas porque Núria tiene alguna duda o porque Xell percibe alguna incorrección. Veamos algunos ejemplos:

(3) 1017. Núria.-Diversió va amb una essa o dues?

1018. Jordi.- Sí, sí. La essa, la essa. 1019. Xell.- Va amb dues.

1020. Núria.-No home, entre la erra i la i!

1021. Jordi.-(Amb to de broma) Molt bé!

Corrigen también algunas palabras. Aunque en el ejemplo siguiente, la corrección no es puramente léxica, sino que supone una revisión conceptual.

(4) 1034. Xell--Jo posaria:... d'altres cultures. Perquè no és veritat d'altres races, és d'altres cultures.

b) El de la lectura final. Una vez pasado «a limpio» el texto, no se origina ningún cambio.

2 La mayoría de revisiones las efectuaron estos alumnos durante el proceso de composición, que como hemos indicado tuvo una duración de 3 horas. Si adoptamos la definición de revisión de Matsuhashi, en el sentido de considerar revisión cualquier operación de reconsideración de lo producido (sea texto escrito o pre-texto según el término que emplea Witte, 1985), y si aceptamos que estas reconsideraciones no llevan necesariamente a introducir cambios en el texto, se comprueba que los alumnos de este grupo efectúan muchas operaciones de revisión. Según el análisis que hemos hecho del total de 86 episodios en que hemos dividido el total de intervenciones de los protocolos, 24 son de 
revisión. Ahora bien, hay intervenciones de revisión insertas en episodios de planificación y de textualización, es decir que el proceso de revisión se aplica recursivamente a diferentes niveles: planificación de contenido, de la estructura del texto, de las frases propuestas para ser escritas, del léxico, etc; y en diferentes momentos a lo largo de todo el proceso. Las intervenciones de revisión suman 292 del total de 1.034 .

3 Como decíamos, la operación de revisión está íntimamente inserta en todos los momentos del proceso de escritura, lo cual confirma su estructura jerárquica y recursiva. En la distribución de episodios por sesiones se observa un cierto predominio de los de planificación en la primera sesión y de los de textualización en la segunda. La mayoría de los de revisión quedan repartidos entre la segunda y la tercera sesión, si bien en esta última ocupan también un lugar importante los de planificación y textualización.

Los dos ejemplos siguientes muestran intervenciones de revisión en episodios de planificación y de textualización, respectivamente.

a) Revisión de la planificación. En el siguiente fragmento de los protocolos los alumnos están considerando la diferencia de trato que se da a los extranjeros según si su origen es europeo (alemanes, ingleses, franceses) o africano (negros, marroquíes, etc). De lo aquí debatido saldrá un párrafo que figura en el borrador pero que no aparecerá, como hemos visto, en el texto definitivo.

(5) 296. Xell.- Què tindran aquells que no tinguin els altres...?

297. Jordi.-Recursos econòmics.

298. Xell.-Sí

299. Jordi.-Va. Però, la contestem?

300. Xell.-Jo no posaria això, perquè...

301. Jordi.-Ho deixem en pregunta?

302. Xell.-Jo no posaria això...

303. Jordi.- $\mathrm{Va}$, va, digues lo teu.
(6) 304. Xell.-Llavors diran: mm... recursos enconòmics.

Clar, i llavors, clar, els negres i els moros $\mathrm{i}$ aquests no tenen recursos econòmics...

305. Núria.-Com ho posem? Ho deixem aixi?

306. Xell.-Poses la pregunta i ja està. 307. Jordi.-Ho deixes amb pregunta? 308. Núria.--Però ells pensaran... 309. Xell.-Ja ho pensaran ells! 310. Jordi.-_Uns diran: un altre color de pell, no?

(...)

313. Xell.-Va.

314. Jordi--No? Un altre color de pell. 315. Xell.-Estem arribant a la conclusió que el racisme és per la pell, és pel color de la pell i no pels recursos econòmics.

La idea predominante de los alumnos hasta ese momento ha sido que las actitudes racistas vienen determinadas principalmente por la falta de recursos económicos de los inmigrantes. Reconsideran ahora lo que han escrito y lo que están proponiéndose escribir, y se dan cuenta de que no se corresponde con la idea inicial, lo cual les conduce a revisarla y reelaborarla. La intervención siguiente de Jordi va en este sentido.

(7) 316. Jordi.-Pels recursos econòmics. Per les dues coses. Que no sempre està relacionat amb l'economia, però normalment sí. Jo què sé.

b) Revisión de la textualización. En el episodio que sigue Xell y Jordi elaboran oralmente el texto que escribe Núria, a la vez que lo van revisando tanto respecto a su contenido como a su forma gramatical. El tema de la discusión entre ellos es complejo. Consideran que históricamente la colonización ha contribuido al desarrollo de nuestro país y en su reflexión se remontan a los inicios de la historia. Como puede comprobarse, sus conocimientos en este campo son más bien escasos.

827. Xell.-(Continua intentant alternatives) Si no ens haguessin ajudat... 
828. Jordi.-Ajudat...

829. Xell.-Que els ibers van ajudar els grecs, no? $\mathrm{O}$ al revés. No me'n recordo.

830. Jordi.-Els ibers estaven aquí ja.

(Reprenen la redacció escrita).

831. Xell.-(Rellegeix) Si a nosaltres no ens...

832. Núria.-(Gairebé alhora)... no

ens... haguessin, no?

833. Xell.-Ajudessin!

834. Núria.-No. No ens...

835. Jordi.-... haguessin...

836. Núria.-Amb bac.?

837. Xell.-Amb bac.

838. Núria.-a..ju ..

839. Xell._... ajudat...

4 Se observa que los alumnos revisan antes de poner el texto por escrito, es decir, hay muchas intervenciones de revisión del pretexto, lo cual da soporte a la hipótesis de Witte sobre la existencia de revisión anterior a la traducción en signos gráficos. La riqueza y variedad de estas revisiones en la situación de redacción en grupo ha planteado la necesidad de distinguir dos tipos de revisión a este nivel. Así, ampliando la propuesta de Witte, distinguiremos dos niveles de revisión anterior a la traducción en signos gráficos: a) Revisión de la planificación, considerada como el subproceso de producción anterior a la linearización del texto en forma sintácticamente estructurada, y que puede afectar tanto al contenido como a aspectos estructurales o retóricos. b) Revisión de lo que denominaremos texto intentado. En los protocolos aparece a menudo un tipo de producciones que aunque son orales constituyen propuestas de texto para ser escrito. Tienen pues las características lingüísticas del texto escrito. Comentaremos a continuación dos fragmentos de las interacciones entre los alumnos que permitirán ejemplificar estos dos tipos de revisión anterior a la producción escrita.

\section{a) Revisión de la planificación.}

Los alumnos hacen una propuesta, en este caso de inclusión en el texto de la definición del término «racista», que han buscado en el diccionario. La discusión tiene dos vertientes: a) la oportunidad de incluir una definición en medio del texto; b) la necesidad de precisar el concepto en función de la audiencia: hay que determinar a qué personas puede considerarse racistas y que constituirán la audiencia a la cual irá dirigida la argumentación. Finalmente la definición no figurará ni en los borradores ni en el texto definitivo. Vemos pues que los alumnos se plantean cuestiones referidas a la estructura textual y a la situación discursiva, y que en este caso reconsideran el texto que van a producir desde este doble punto de vista.

(9) 73. Xell.-(disposada a dictar a la Nùria que escriu) Quan parlem de racisme... O quan es parla de racisme... és justificar.. el fet de que altres races o cultures siguin sotmeses a l'explotació. 74. Jordi-_Això ara? No ficaràs una definició enmig del text.

75. Xell.-Per què no?

76. Jordi.-Val, no aquí, ara.

77. Xell.--Per què no? Hi ha gent que es pensa que és racista i no ho és. Es una altra cosa.

78. Jordi.-Però també ho has d'introduir d'alguna manera.

79. Xell.-Si l'introduïm. Mira: «Quan parlem del racisme...» és una introducció. Justifica el fet de que...

80. Núria.-Fiquem això ara? Es que jo no ho entenc aquí. Que cadascú tingui la seva opinió sobre el racisme. Si algú es pensa que és una cosa, cadascú té una opinió.

b) Revisión del texto intentado (Ti). Muchas veces los alumnos, antes de escribir una frase o un fragmento cualquiera de texto, la formulan oralmente. Esta formulación es a menudo evaluada por el mismo que la ha formulado o por algún otro miembro del grupo. De esta evaluación se sigue muchas veces algún cambio en la frase propuesta o el abandono de la propuesta y la formulación de otra frase. Algunos rasgos permiten diferenciar estas intervenciones de las que son exclusivamente de planificación:

1) Tienen lugar después de expresiones co- 
mo: «pongamos», «venga, digamos ahora», «podemos decir», «metamos», etc.

2) Hay cambio de entonación en la intervención que marca de manera clara la diferencia entre las intervenciones en que discuten algún elemento de la planificación y estas producciones formuladas para ser escritas.

3) Hay un cambio en el registro lingüístico: tono más impersonal, apelación a los receptores, etc. En general se podría decir que las características lingüísticas del $T i$ son las propias del texto escrito (Te) de los mismos alumnos y difieren en cambio de las otras producciones orales.

¿En el ejemplo siguiente transcribimos en negritas las intervenciones que corresponden a lo que llamamos texto intentado (Ti).

(10) 443. Jordi.-Però ningú vol fer aquestes feines.

444. Xell.-Però qui vol cobrar mil pessetes al día...

445. Núria.-I els emigrants, com que només tenen aquest remei, les accepten.

446. Núria.- (Escrivint) Els emigrants...

447. Núria.-(S'autocorregeix) Com que no tenen cap més remei i...

448. Xell.-Però els emigrants. . tampoc ho volen fer.

449. Núria.-Però com que no tenen

cap més remei i...

no tenen estudis per fer altres feines... han de fer aquestes que són les úniques que.. saben fer.

450. Jordi.--Però per desgràcia per ells, no?

451. Xell._... tampoc ho poden fer però... con que...

452. Núria.-Els emigrants...

453. Jordi.-(Corregint el que ha dit la Xell) Ho volen.

454. Xell.-... ho volen...

455. Jordi.-... però no tenen cap més remei ni...

456. Xell.-... però...

457. Jordi.-... estudis per fer altres feines.
458. Xell.-No tenen més coneixements.

459. Jordi.-No tenen coneixements, més coneixements. Més coneixements.

460. Núria.-... i accepten lo primer que els hi donen.

461. Jordi.-... més coneixements per fer altres feines $i$ accepten.. (Com si dictés) Per... fer altres feines...

Las tres primeras intervenciones del ejemplo $(443,444$ y 445$)$ son tres propuestas distintas que cada alumno hace casi simultánamente. Parece que cada uno siga su camino sin atender a los demás. Pero Xell se pone a escribir la frase corrigiendo su propuesta anterior; es decir, revisa su propio texto intentado. A continuación (448) Xell hace un nuevo cambio e inserta una nueva idea. En la intervención 449 Núria empieza repitiendo la frase que ha propuesto con la entonación propia del Ti para cambiar de entonación y pasar a proponer un contenido que no parece tener la pretensión de ser escrito tal cual. Así lo interpreta Jordi, que en la intervención 450 comenta lo dicho por Núria. Mientras tanto, Xell sigue escribiendo de acuerdo con la idea iniciada en 446. En la intervención 453 Jordi introduce una corrección en el Ti (en vez de «ho poden» propone escribir «ho volen») que se acepta sin discusión y en la 458 Xell introduce otra aceptada también por todos (cambia «studis» por «més coneixements»).

En la intervención 73, transcrita más arriba, podemos ver una autocorrección del texto que está proponiendo para que sea escrito. En ella vemos claramente el cambio de un lenguaje más implicado por el uso de la primera persona, aunque tenga en este caso un valor generalizador, al uso de la forma impersonal con se ( ... es parla de racisme...») más distanciada y por lo tanto considerada más propia del lenguaje escrito.

En la situación de elaboración colectiva del escrito es, en algunos casos, difícil diferenciar las intervenciones de $\mathbf{T i}$ de las producciones que acompañan la acción de escribir sean las vocalizaciones que del mismo que es. tá escribiendo, sean las del compañero que 
está dictando o en algunos casos leyendo lo que el otro escribe, y que, por lo tanto, pueden ser ya consideradas de producción de texto escrito. De hecho, y hablando en sentido estricto, todas las intervenciones de textualización que aparecen en los protocolos o bien son texto que debe escribirse (algunas de ellas como una especie de dictado o autodictado) o lectura del texto ya escrito. De todos modos, creemos interesante diferenciar las vocalizaciones que acompañan la escritura, a las que llamamos intervenciones de textualización de Te, de las intervenciones que se formulan en forma de propuesta sin que acompañen de manera directa a la acción de escribir. La impresión es que el Ti se formula para ser reconsiderado, evaluado por todos los miembros del grupo antes de que se tome la decisión de ponerlo por escrito.

Este planteamiento acepta que las operaciones de elaboración líneal del texto constituyen el subproceso de textualización (o linearización según Bronckart y Schneuwly) y no de planificación, reservando este término para todas las operaciones anteriores a la configuración sintáctica de los enunciados. Así pues, en situación de redacción en grupo algunas producciones orales contituyen ya elementos linearizados y preparados para ser escritos. Esta constatación no presupone que en la producción individual se den operaciones de linearización anteriores a la puesta por escrito del texto, aunque tampoco la excluye. Creemos, en cambio, que es un elemento característico de la situación de escritura en grupo.

5. Las operaciones de revisión efectuadas durante el proceso no conducen siempre a efectuar cambios en el Te o en el $\mathbf{T i}$, lo cual queda explicado en el modelo de Bereiter y Scardamalia $(1983,1987)$. En muchas ocasiones alguno de los alumnos percibe un desajuste que por motivos varios (incapacidad de diagnosticar el problema, falta de recursos linguísticos o discursivos, etc.) el grupo no puede resolver.

(11) 857. Núria.-(Rellegeix la darrera frase que ha escrit) «Es veritat que hi ha algunes cultures menys desenvolupa- des que altres perquè això tenim...» (S'adona que el que diu no funciona. Encara que no és el que hi ha escrit, la mala lectura els porta a fer canvis en el text).

858. Jordi._... però per això hem de (posa èmfasi en la forma normativa d'obligació) (Reprèm la frase) Però per això no els tenim...

859. Xell.-Encara serà racista!

860. Jordi.-.. per això no el tenim... per això els tenim...

861. Jordi.-(Llegeix la frase següent) «Us molesta ajudar a les altres races? Si no ens haguessin ajudat encara estariem al Neolític».

Jordi compara las dos estructuras: «... per això els tenim d'ajudar..." $y$ «... per això no els tenim d'ajudar...» sin decidirse en esta ocasión por ninguna de las dos. La dificultad de una posible doble negación («no per això els hem de deixar d'ajudar»o como escriben finalmente) le impide encontrar la solución adecuada. Posteriormente lo resolverá Xell directamente en el borrador sin hacer ningún comentario oral, aunque su solución mantiene un uso no adecuado de la conjunción «per això» (por eso). En el borrador se ve además la corrección parcial de la forma de obligación que en catalán normativo es «hem de...» y no «tenim que...».

6. El desencadenante de una operación de revisión no siempre es la percepción de un desajuste, de una disonancia entre intención y producción (sea planificación o texto). $\mathrm{Pa}$ rece más bien que en este grupo las operaciones de revisión son consecuencia de una actitud que orienta a los alumnos a evaluar $y$, por tanto, a reconsiderar constantemente su producción. En algunas ocasiones esta evaluación los lleva a darse por satisfechos. Por tanto, no se puede situar el inicio de la revisión en la percepción de un desajuste, de una disonancia; revisar es una operación inherente al proceso de producción escrita que puede estar motivada por causas distintas, entre las que, a modo de ejemplo, podemos citar:

a) Percepción de un desajuste, de una disonancia. 
b) Pérdida de control de la producción en su conjunto, o de alguna de sus partes (frase, párrafo).

c) Recuperación de las ideas expresadas para proseguir la redacción enlazando con las nuevas.

Este planteamiento se vincularía con un concepto de revisión no relacionado sólo con la percepción de disfunciones, sino como un proceso que conduce a la evaluación del texto producido y de la representación tanto del texto ya escrito como del que se quiere intentar. (Matsuhashi, 1987).

7. Los estudiantes de este grupo revisan a todos los niveles. Algunos ejemplos ilustran claramente que estos alumnos reconsideran su escrito a niveles globales, y no sólo en relación con los contenidos, sino también respecto a la estructura y la función del texto.

a) Revisión de la función del texto. Los alumnos tienen claro desde el inicio del trabajo que la función del texto que escriben es convencer a una posible audiencia. Xell, que tiene una actitud extraordinariamente crítica respecto del texto que están produciendo tienen la impresión que es poco convincente y así lo plantea al resto del grupo. Lo que ha escrito, que no figurará en el texto definitivo, es lo siguiente (ver figura 1).

\section{(Borrador)}

Para «poner a prueba» su texto, Xell trata de que Jordi asuma el papel de receptor. Como no lo consigue porque Jordi no entiende lo que ella pretende, ella misma se desdobla y establece un diálogo entre el texto y un posible receptor con ideas racistas, para comprobar si hay argumentos más fuertes que su argumentación. El resultado la deja bastante insatisfecha.

(12) 507. Xell.-No sé, no m'acaba de convèncer.

508. Jordi.-El què, lo del sou?

509. Xell.-Tot el text.

510. Jordi.-Jolin!

511. Xell.-Es que mira (Rellegeix el que han escrit): «Quan ve gent d'altres cultures al nostre país, ens treuen els llocs de treball; però, qui vol cobrar mil pessetes al día treballant deu hores diàries? Els emigrants tampoc ho volen fer però no tenen més coneixements per fer altres feines, per això han de fer les feines que saben fer; però per desgràcia són les més mal pagades». A qui, a qui conveç?

512. Jordi.-Home...!

513. Xell.-Tu sigues racista, eh? Ara ets racista i dius: Fora els negres perquè són... no tenen coneixement de res.. ens prenen els llocs de treball... 514. Jordi.-Que ells tampoc no ho volen fer.

Xell se da cuenta que Jordi no ha entendido lo que pretende y continúa ella sola.

515. Xell--Pero jo sóc un racista que trobo que els negres són uns estúpids, que no saben fer res i que treuen els llocs de treball i que, com diu aquí, no se'ls hauria de pagar. (Inicia una mena de diàleg entre el que diu el text que ha escrit i ella com a hipotètica racista).

- «Quan ve gent d'altres cultures al nostre pais ens treu el lloc de treball». -Clar.

- «Però, qui vol cobrar mil pessetes al dia treballant deu hores?»

-Encara gràcies que les cobren!

516. Núria.-Què? (No estava atenta al diàleg entre la Xell i el Jordi i no entén què estan fent).

517. Xell.-(Continuant el seu diàleg hipotètic)

-Encara gràcies que les cobren.

- «Els emigrants tampoc ho volen fer».

-Doncs que no vinguin.

- «Però no tenen més coneixements

per fer altres feines».

-Es que són tontos.

-No saben fer res.

- «Per això agafen les feines que saben fer».

- No saben fer res.

- «Però per desgràcia són les més mal pagades». 
-Es que no els haurien de pagar. 518. Jordi.-Però és que en tots els textos també pots fer això.

519. Xell.-Ja, però...

520. Núria.-Això ens passaria a tot arreu.

521. Xell.-No sé.

522. Jordi-Un racista, si ell no es vol treure la idea, pues...

523. Xell.-Ja, però... això no convenç gaire.

524. Jordi._... trobarà tots els punts que està malaments, doncs.. que es foti, que se'n vagi a la merda.

525. Xell.-Sí, però...

La riqueza de este fragmento permitiría numerosos comentarios, como por ejemplo sobre la conclusión a la que llega Jordi sobre la dificultad de convencer a los no convencidos, y por lo tanto la constatación de que a menudo la argumentación se dirige a enriquecer las opiniones de aquellos que ya comparten de algún modo la opinión que se defiende. Como el presente artículo se centra en el comentario de las operaciones de revisión no profundizaremos en este aspecto referido a la definición de la audiencia.

b) El ejemplo siguiente muestra una secuencia de revisión de los contenidos expresados en el texto ya escrito. Xell evalúa lo que han escrito y saca la impresión de que el texto no dice exactamente lo que ellos han querido decir. Esta constatación la lleva a considerar que hace falta un nuevo contenido: un contraargumento, como ella dice, que «se lo lleve todo aguas abajo».

(13) 431. Núria.-I després posa: I quines feines!

432. Xell.—No, però... jo diria que.. això..., vull dir (Rellegeix de pressa $\mathrm{i}$ en veu molt baixa):

«però qui vol cobrar mil pessetes al dia per treballar deu hores diàries?» (Es queda com en suspens) Ara ho corregirem bastant tot, perquè estem deixant...

433. Núria._... els negres fets pols. 434. Xell.-(Rient) Síiii. Perquè vo- lem dir que... que els negres volen cobrar mil pessetes per treballar deu hores diàries.

435. Núria.-Es clar!

436. Xell.-Això... (Amb èmfasi) hemn de fer un bon contraargument que... s'endugui tot riu avall, en!

c) Las intervenciones 74 a 80 transcritas antes son un buen ejemplo de revisión de la estructura del texto en relación con las necesidades de la posible audiencia: La conveniencia de introducir la definición del término «racista» viene determinada, en opinión de Xell, por la necesidad de delimitar a quiénes se dirige el texto; define, por lo tanto, los posibles interlocutores de la argumentación.

\section{CONCLUSIONES}

De lo dicho hasta ahora pueden sacarse algunas conclusiones que deberían considerarse más bien hipótesis para futuras investigaciones centradas en el análisis del proceso de redacción de los alumnos.

Destacaremos en primer término las que hacen referencia al proceso de composición. En la situación de redacción en grupo nuestras observaciones dan soporte a la hipótesis de Witte en el sentido que hay revisión anterior a la plasmación del texto por escrito. Los alumnos del grupo observado realizan numerosas revisiones pre-textuales, muy superiores en número y calidad a las que realizan en el texto ya escrito. Es precisamente la riqueza y variedad de estas revisiones lo que nos ha llevado a la necesidad de distinguir, de momento, dos niveles de revisión pre-textual: a) revisión de la planificación, considerada como el subproceso de elaboración pre-lineal del texto, y b) la revisión de lo que hemos dado en llamar texto intentado. La situación de redacción en pequeño grupo provoca la aparición oral de este tipo de producciones que tienen la forma sintáctica propia del texto escrito y que en su producción oral se distinguen por la entonación con que son pronunciadas y por los elementos de introducción que las anuncian. Estas producciones 
son evaluadas y revisadas por uno o más miembros del grupo antes de ponerlas por escrito, lo cual les lleva a efectuar cambios o a rechazarlas e intentar un nuevo texto.

En segundo lugar se pone de manifiesto no sólo la recursividad del proceso, sino también su carácter jerárquico. En cada episodio en que hemos dividido las producciones orales registradas en los protocolos hay una operación dominante, pero en cada una de ellas están insertas otras operaciones. $Y$ es especialmente la de revisión la que se interrelaciona con todas las demás en cada momento de la producción.

En tercer lugar, destaca el hecho de que, pese a confirmarse aparentemente que los estudiantes hacen pocos cambios en el texto terminado o en el último borrador, en cambio los alumnos observados revisan mucho durante el proceso de producción y a todos los niveles, desde la ortografía hasta la misma función del texto. Muchas de estas operaciones de revisión, como hemos dicho, no se traducen en cambios en el texto escrito.

Es posible que estos resultados sean atribuibles a la especificidad de la situación de producción en grupo. A diferencia de muchas de las investigaciones sobre la revisión por parejas o por grupo que se han llevado a término, estas observaciones se hacen sobre el proceso mismo de producción, lo cual permite por un lado tener acceso a las revisiones que de manera espontánea se plantea el grupo y que no han sido inducidas por el profesor $o$ el experimentador, y por otro observar la revisión pretextual a la cual es imposible acceder cuando el trabajo que se pide a los alumnos parte de un texto ya escrito que hay que revisar. Conocemos pocas investigaciones que analicen estos procesos y creemos que es un camino que permitiría conocer de qué forma los aprendices se plantean los problemas de los diferentes tipos de textos escritos y sobre las diferentes situaciones discursivas en que estos se utilizan.

Por lo que se refiere a posibles conclusiones sobre el proceso de aprendizaje, el trabajo sugiere más preguntas que respuestas. Primeramente, llama la atención que no haya relación entre la calidad del escrito final y la cantidad y calidad de intervenciones de revisión que hacen los alumnos observados. El texto final producido tiene muchas deficiencias tanto de coherencia global como de estructura. Sorprende especialmente el hecho de que abandonen alguno de los temas más discutidos a lo largo del proceso de producción. Esto sugiere que la evaluación del aprendizaje de la redacción en la escuela no puede basarse únicamente en los textos producidos, sino que habría que tener en cuenta los procesos seguidos por los alumnos y las dificultades de dichos procesos según el tipo de texto. En general el juicio de los profesores sobre los textos de sus alumnos tiene más en cuenta la capacidad de manetener la fluidez que el dominio de las condiciones de escritura más complejas o el intento de ajustarse a ellas. Como dice Applebee (1984), «un estudiante que hace un esfuerzo real, pero infructuoso, de utilizar una estructura analítica resulta de hecho penalizado frente a otro que evita la dificultad y adopta un modelo más familiar». El grupo de Xell, Núria y Jordi aborda la tarea tomando en consideración todos los elementos que la hacen problemática. En los protocolos se ve claramente que son conscientes de los problemas que han de resolver y que hablan de ellos. Pero no llegan del todo a buen puerto: el texto «no convence». Entre los grupos que hicieron el mismo trabajo, hay algunos, en cambio, que siguieron una senda más llana sin que el resultado fuera peor; al contrario, en algunos casos el texto es más fluido y se lee más faćilmente. Habría que preguntarse si los alumnos del grupo analizado, al verbalizar los problemas y abordarlos, han aprendido, aunque este aprendizaje no se manifieste inmediatamente, y en qué condiciones les permitirá aprendizajes posteriores con resultados más fecundos. Sería de esperar que en algunos casos hubiera diferencias entre estos alumnos $\mathrm{y}$ aquellos que no se han planteado siquiera los problemas. Y aquí se abre un nuevo interrogante: $i s o n$ las condiciones especiales del trabajo las que llevan a revisar? Nuestra hipótesis es que sí, que la dificultad de la tarea por un lado y la necesidad de compartirla por otro provocan la aparición de las operaciones 
77

de revisión. Habría que saber, sin embargo, qué características de la colaboración del grupo lo han hecho posible; y también hasta qué punto han incidido los rasgos personales de uno de los miembros del grupo. Y habría que saber también si lo que han aprendido a ha- cer en grupo se transferirá posteriormente al proceso individual. La investigación en el campo de la didáctica debería abordar estas cuestiones que creemos cruciales para un adecuado planteamiento de la enseñanza de la composición escrita.

quan be gend d'altea cultures al mostro païs ens trewen els laces de treball... Pero qui wol cabrar 1000 pts al dia tedballant deu hares diaries)

Els emmigrants tampoc ho poden fer, paro noteras mias coneixaments per fer altees faimes, per això agafen lis fines que desgracia sionles mes mal pagades.

Els conos

Figura 1

Borrador del texto de Xell

Notas

1 La maestra considera que Xell escribe bien y es una buena alumna, creativa, con ideas que se reflejan en lo que escribe.

2 Todos los protocolos se citan en catalán. La traducción va en apéndice al presente artículo.

Referencias

Applebee, A.N. (Ed.) (1984). Context for Leaming to Write: Studies of Secondary School Instruction. Norwood, N. J.: Ablex.

BartuETt, E.J. (1982). Learning to revise: Some component processes. A Nystrand, M. (Ed.): What Writers Know. Nueva York: Academic Press, 345-363.

Bereiter, C., y Scardamalia, M. (1987). The Psycbology of Written Composition. Hillsdale, NJ.: Erlbaum.

BRIDWELL, L. S. (1980). Revising strategies in twelfth grade students' transactional writing. Research in the Teaching of English, 14, 197-222. 
BRITTON, J. (1987). Writing and Reading in the Classroom. Center for the Study of Writing, Technical Report, 8. Berkeley: University of California y Pittsburg: Carnegie Mellon University.

Calkins, L. Mc. (1986). The art of Teaching Writing. Portsmouth, NH.: Heinemann.

CAMPS, A. (1989). Modelos del proceso de redacción: algunas implicaciones para la enseñanza. Infancia y Aprendizaje, 49, 3-19.

Camps, A. (1990). Models del procés i ensenyament de la redacció. A Camps, A., et al.: Text $i$ ensenyament. Una aproximació intendisciplinària. Barcelona: Barcanova. 11-29.

Cassany, D. (1989). La correcció del treball escrit. Vic: Eumo.

Cooper, CH. R., y Matsumashi, A. (1983). A theory of the writing process. A Martlew, M. (Ed.): The Psychology of Written Language. Nueva York: John Wiley and Sons.

EMIG, J. (1971). The Composing Process of Twelfth Graders. (Research Report n. 13). Urbana, Ill.: National Council of Teachers of English.

Flanigan, M., y MenÉndez, D. S. (1980). Perception and change: Teaching revision. College English, 43 (3), $256-266$.

Flower, L., y Hayes, J. R. (1980). Writing as a problem solving. Visible Language 14, 4, 388-399.

Flower, L., y HAYEs, J.R. (1981). The cognition of discovery: Defining a rhetorical problem. College Composition and Communication, 31, 21-32.

Forman, E. A., y CAzDEn, C. B. (1984). Perspectivas vygotskianas en la educación: el valor cognitivo de la interacción entre iguales. Infancia y Aprendizaje, 27-28, 139-157.

Freedman, S. W. (Ed.) (1985). The Acquisition of Written Language. Response and Revision. Norwood, NJ.: Ablex.

Garcia.Debanc. C. (1984). Une évaluation formative en pédagogie de l'écriture. Pratiques, 44, $21-52$.

García.Debanc, C., y Mas, M. (1987). Evaluation des productions écrites. A Chiss, J.-L., et al. (Ed.): Apprendre/Enseigner à produire des textes écrits. Bruxelles: De Boeck, 133-145.

Gere, A. R., y Stevens, R. S. (1985). The language of writing groups: How oral response shapes revision. A Freedman, S. W. (Ed.): The Acquisition of Written Language. Norwood, NJ.: Ablex, 85-105.

Graves, D. H. (1979). What children show us about revision. Language Arts, 55, 393-399.

Graves, D. H. (1983). Writing. Teachers and Children at Work. Portsmouth, NH.: Heinemann. (Trad. castellana: Didáctica de la escritura. Madrid: MEC/ Morata.)

Hayes, J. R., y Flower, L. (1980). Identifying the organization of writing process. A Gregg, L.W., y Steinberg, E. R. (Eds.), Cognitive Processes in Writing. Hillsdale, N. J.: Lawrence Erlbaum, 3-30.

Hillocks, G. (1986). Research on Written Composition. New Directions for Teaching. Urbana, Ill.: National Conference on Research in English.

Matsuhashi, A. (1982). Explorations in the real-time production of written discourse. A Nystrand, M. (Ed.): What Writers Know. Nueva York: Academic Press, 269-290.

Matsuhashi, A. (Ed.) (1987a). Writing in Real Time. Norwood, NJ.: Ablex.

Matsuhashi, A. (1987b). Revising the plan and altering the text. A Matsuhashi, A. (Ed.): Writing in Real Time. Norwood, NJ: : Ablex, 197-223.

Matsumashi, A., y Gordon, E. (1985). Revision, addition, and the power of the unseen text. A Freedman, S. W. (Ed.): The Acquisition of Written Language. Norwood, NJ.: Ablex, 226-249.

Murray, D. M. (1978). Internal revision: A process of discovery. En C.R. Cooper y L. Odell (Eds.), Reseach on Composing: Points of Departure. Urbana, Ill.: National Council of Teachers of English, 85-103.

PERL, S. (1979). The composing process of unskilled college writers. Research in the Teaching of English, 13, 317-356.

PiAzZA, C. L. (1987). Identiying context variable in research on writing. Written Communication, 4 (2), $107-137$.

Pianko, S. H. (1979). A description of the composing processes of college freshman writers. Reseanch in the Teaching of English, 13, 5-22.

Scardamalis, M., y Bereiter, C. (1983). The development of evaluative diagnostic, and remedial capabilities in children's composing. A Martlew, M. (Ed.): The Psychology of Written Composition, Nueva York: John Wiley and Sons.

Sommers, N. (1980). Revision strategies of student writers and experienced adult writers. College Composition and Communication, 31, 378-388.

Sommers, N. (1982). Responding to student writing. College Composition and Communication, 33 (2), $148-156$.

STALLARD, L. M. (1974). Analysis of the writing behavior of good student writters. Research in the Teaching of English, 8, 206-218.

WITTE, S. P. (1985). Revising, composing theory, and research desing. A Freedman, S. W. (Ed.): The Acquisition of Written Language. Norwood, NJ.: Ablex, 250-285.

\section{Anexo}

(1) 134. Xell._ ¡Un texto normal! Yo quisiera hacer un texto.

(2) -El mundo está lleno de animales que se matan el uno al otro para sobrevivir uno mismo y jamás por diversión ni por el gusto de matar, esto lo hace un solo animal de los miles que existen. El hombre mata para sentirse superior, para ser el mejor y tener poderes.

Cuando se enteraron de que había otras culturas fue cuando empezó a aparecer el racismo, pero entonces nuestra mente no estaba tan desarrollada como ahora, ya es hora de que cambiemos. 
¿Por qué la gente de culturas más bajas están marginados en nuestra sociedad? Es cierto que algunas culturas están menos desarrolladas que otras, pero no por esto tenemos que rechazarlos, sino ayudarlos; hacer escuelas, campos de trabajo, etc.

el «Neolítico».

¿ Os molesta ayudar a las otras razas? $\mathrm{Si}$ a nosotros no nos hubiesen ayudado aún estaríamos en

¿Tienen derecho a quitarnos los puestos de trabajo? Esta gente que emigran a otros países tienen la misma necesidad que nosotros tuvimos hace unos cuantos años, en la época del franquismo. ¿Por qué no podemos ayudarles ahora?

«El mundo es de todos y hemos de compartirlo».

(3) 1017. Núria.-¿«Diversió» va con una ese o con dos?

1018. Jordi.-Sí, sí. La ese, la ese.

1019. Xell.-Va con dos.

1020. Núria.-iNo, hombre, entre la erre y la i!

1021. Jordi. - (En tono de broma) ¡Muy bien!

(4) 1034. Xell.-Yo pondría:... de otras culturas. Porque de otras razas no es verdad, es de otras culturas.

(5) 296. Xell.-¿Qué tendrán esos que no tengan los otros...?

297. Jordi.-Recursos económicos.

298. Xell.-Sí.

299. Jordi.-Va. Pero ¿la contestamos?

300. Xell.-Yo no pondría esto, porque..

301. Jordi.- ¿Lo dejamos en pregunta?

302. Xell.-Yo no pondría esto...

303. Jordi.-Venga, venga, di lo tuyo.

(6) 304. Xell -_Entonces dirán: mm... recursos económicos.

Claro, y entonces, claro, los negros y los moros y ésos no tienen recursos económicos...

305. Núria.- ¿Cómo lo ponemos? ¿Lo dejamos así?

306. Xell - Pones la pregunta y ya está.

307. ¿Lo dejas como pregunta?

308. Núria.-Pero ellos pensarán...

309. Xell - ¿ $\mathrm{Ya}$ lo pensarán ellos!

310. Jordi.-Unos dirán: otro color de la piel, ¿no?

(...)

313. Xell. $-\mathrm{Va}$.

314. Jordi. - ¿No? Otro color de la piel.

315. Xell.-Estamos llegando a la conclusión de que el racismo es por la piel, es por el color de la piel y no por los recursos económicos.

(7) 316. Jordi.-Por los recursos económicos. Por las dos cosas. Que no siempre está relacionado con la economía, pero normalmente sí. Yo qué sé.

(8) 827. Xell.-(Sigue ensayando alternativas) Si no nos hubiesen ayudado...

828. Jordi.-Ayudado...

829. Xell.-Que los íberos ayudaron a los griegos, ¿no? O al revés. No me acuerdo.

830. Jordi.-Los íberos estaban aquí ya.

(Reanudan la redacción escrita).

831. Xell.-(Relee) Si a nosotros no nos...

832. Núria.- (Casi al mismo tiempo)... no nos... hubiesen, ¿no?

833. Xell.-_Ayudasen!

834. Núria.-No. No nos...

835. Jordi.-... hubiesen...

836. Núria.-¿Con hache?

837. Xell.-Con hache.

838. Núria,-a... yu...

839. Xell.-... ayudado...

(9) 73. Xell.-(Dispuesta a dictar a Núria, que escribe). Cuando hablamos de racismo... O cuando se habla de racismo... es justificar... que otras razas o culturas estén sometidas a explotación.

74. Jordi.- ¿Esto aquí? No meterás una definición en mitad del texto.

75. Xell.-_Por qué no?

76. Jordi.-Vale, no aquí, ahora.

77. Xell.- ¿Por qué no? Hay gente que piensa que es racista y no lo es. Es otra cosa.

78. Jordi.-Pero también lo has de introducir de alguna manera.

79. Xell.-Si lo introducimos. Mira: «Cuando hablamos del racismo...» es una introducción. Justifica que...

80. Núria.-_ ¿Metemos esto ahora? Es que yo aquí no lo entiendo. Que cada uno tenga su opinión sobre el racismo. Si alguien piensa que es una cosa, cada uno tiene una opinión.

(10) 443. Jordi.-Permo nadie quiere hacer estos trabajos.

444. Xell.-Pero quién quiere cobrar mil pesetas al día...

445. Núria.-Y los emigrantes, como no tienen ningún otro remedio, las aceptan.

446. Xell.-(Escribiendo) Los emigrantes...

447. Núria.-(Se autocorrige) Como no tienen más remedio y...

448. Xell.-Pero los emigrantes... tampoco quieren hacerlo. 
449. Núria.-Pero como no tienen más remedio y... no tienen estudios para hacer otros trabajos... han de hacer éstos, que son los únicos que saben hacer.

450. Jordi.-Pero por desgracia para ellos, ¿no?

451. Xell. -... tampoco pueden hacerlo pero... como que...

452. Núria.-Los emigrantes...

453. Jordi.-(Corrigiendo lo que ha dicho Xell) Lo quieren.

454. Xell. -... lo quieren...

455. Jordi._... pero no tienen más remedio ni...

456. Xell.-... pero...

457. Jordi.-... estudios para hacer otros trabajos.

458. Xell.-No tienen más conocimientos.

459. Jordi.-No tienen conocimientos, más conocimientos. Más conocimientos.

460. Núria.-... y aceptan lo primero que les dan.

461. Jordi.-... más conocimientos para hacer otros trabajos y aceptan... (Como si dictase) Para... hacer otros trabajos...

(11) 857. Núria.-(Relee la última frase que ha escrito) «Es verdad que hay algunas culturas menos desarro-

lladas que otras porque esto tenemos...»

(Se da cuenta de que lo que dice no funciona.

Aunque no es lo que está escrito, la mala lectura los lleva a hacer cambios en el texto).

858. Jordi._... pero por esto hemos de (pone énfasis en la forma normativa de obligación) (Retoma la frase). Pero por esto no los tenemos...

859. Xell.-iAún será racista!

860. Jordi.-... por esto no lo tenemos... por esto los tenemos...

861. Jordi.-(Lee la frase siguiente) « $\mathrm{O}$ Os molesta ayudar a las otras razas? Si no nos hubiesen ayudado todavía estaríamos en el Neolítico».

(12) 507. Xell.-No sé, no acaba de convencerme.

508. Jordi.-¿Qué, lo de la paga?

509. Xell.-Todo el texto.

510. Jordi.-iJolín!

511. Xell.-Es que mira (relee lo que han escrito): «cuando viene a nuestro pais gente de otras culturas, nos quitan los puestos de trabajo: pero cquién quiere cobrar mil pesetas al día trabajando diez horas diarias? Los emigrantes tampoco quieren hacerlo, pero no tienen más conocimientos para hacer otros trabajos, por esto han de hacer los trabajos que saben hacer; pero por desgracia son los peor pagados». ¿A quién, a quién convence esto?

512. Jordi.

513. Xell.-Tú sé racista, ¿eh? Ahora eres racista y dices: Fuera los negros porque son... no tienen conocimientos de nada... nos quitan los puestos de trabajo...

514. Jordi.-Que ellos tampoco quieren hacerlo.

515. Xell.-Pero yo soy un racista y encuentro que los negros son unos estúpidos, que no saben hacer nada y quitan los puestos de trabajo y que, como dice aquí, no habría que pagarles (inicia una especie de diálogo entre lo que dice el texto que han escrito y ella como hipotética racista):

- «Cuando viene gente de otras culturas a nuestro país nos quita el puesto de trabajo».

-Claro.

- «Pero ¿quién quiere cobrar mil pesetas al día trabajando diez horas?»

- iAún gracias que las cobran!

516. Núria.—¿Qué? (No estaba atenta al diálogo entre Xell y Jordi y no entiende lo que están haciendo)

517. Xell.-(Continúa el diálogo)

-Aún gracias que las cobran.

- «Los emigrantes tampoco quieren hacerlo.»

-Pues que no vengan.

- «Pero no tienen más conocimientos para hacer otros trabajos.»

-Es que son tontos.

- «Por esto toman los trabajos que saben hacer.»

-No saben hacer nada.

- «Pero por desgracia son los peor pagados.»

-Es que no les tendrían que pagar.

518. Jordi.-Pero es que con todos los textos puedes hacer esto.

519. Xell.-Ya, pero...

520. Núria.-Esto nos pasaría en todas partes.

521. Xell.-No sé.

522. Jordi.-Un racista, si él no se quiere quitar la idea de la cabeza, pues...

523. Xell.-Ya, pero... esto no convence demasiado.

524. Jordi._... encontrará todos los puntos que están mal, pues... peor para él, que se vaya a la mierda.

525. Xell.-Sí, pero...

(13) 431. Núria. $-\mathrm{Y}$ después pon: $\mathrm{i} Y$ vaya trabajos!

432. Xell.-No, pero... yo diría que... esto..., quiero decir (relee deprisa y en voz muy baja): «pero ¿quién quiere cobrar mil pesetas al día por tabajar diez horas diarias?》 (se queda como en suspenso). Ahora lo corregiremos bastante todo, porque estamos dejando..

433. Núria - _... a los negros hechos polvo. 
434. Xell.-(Riéndose) Síiii. Porque queremos decir que... que los negros quieren cobrar mil pesetas por trabajar diez horas diarias.

435. Núria. ${ }_{\text {CClaro! }}$

436. Xell.-Eso... (con énfasis), hemos de hacer un buen contraargumento que... se lo lleve todo aguas abajo, jeh!

\section{Extended Summary}

This paper first reviews some of the most significant research on revision processes, from those carried out in the 70's focusing in modifications in the written text (e.g., Sommers, 1979; Bridwell, 1980), to the formulation of most recent models discussing the potential components of this process. Some of the most important contributions concerning revision processes are the following: a) it is seen as hierarchical and recurrent (Hayes and Flower, 1980); b) it involves complex operations (e.g., Bartlett, 1982; Bereiter and Scardamalia, 198); c) it may result in no change in the written text and bear only on pre-textual elements (Bereiter and Scardamalia, 1987; Witte, 1989; Matsuhashi, 1987).

In the second part, the protocols from the oral interactions between three 13-14 years old students writing in group an argumentative text are discussed. This allow us to offer some comments on the composition processes of novice writers in a group situation. The most salient findings refer to: a) the number and quality of the revision operations orally carried out in contrast with the reduced number of revision operations reflected in the written text; b) the small size and bad quality of the final text. In this respect, two different kinds of pre-textual revisions have been observed: i) revisions which have an effect on text planning, and ii) revisions which have an effect on productions, stated as proposals to be written down, which we consider to be part of the textualization subprocess. The latter would include two kinds of productions: those to the intended text (oral productions), and those related to the written text (productions in graphic form).

Furthermore, against the general notion that students revise only at superficial level, it was observed that students' intervened in the revision of global aspects in numerous and very complex, ways. The need to revise seems more motivated more by a general attitude of reconsidering production than by a simple perception of non adjustment between what they do write and what they would like to write.

The hypothesis is put forth that this situation of working in a group may be the triggering factor that causes the revision operations to appear. At least it seems clear that the needs resulting from working together compels students to state revisions explicitly. However, the question about the transference of these operations to the individual processes remains open.

Finally, the need to carry out further research on students' production processes within the school context is raised. The aim being to further our knowledge and evaluate the problems involved for the students togetther with the strategies they use in trying to solve them. This would improve interventions and provide a guidance in this activity. 